\title{
The Integrating Factors for Riccati and Abel Differential Equations
}

\author{
Chein-Shan Liu ${ }^{1}$ \\ ${ }^{1}$ Department of Civil Engineering, National Taiwan University, Taipei 106-17, Taiwan \\ Correspondence: Chein-Shan Liu, Department of Civil Engineering, National Taiwan University, Taipei 106-17, \\ Taiwan. E-mail: liucs@ntu.edu.tw
}

Received: March 20, 2015 Accepted: April 10, 2015 Online Published: May 2, 2015

doi:10.5539/jmr.v7n2p125 URL: http://dx.doi.org/10.5539/jmr.v7n2p125

\begin{abstract}
We can recast the Riccati and Abel differential equations into new forms in terms of introduced integrating factors. Therefore, the Lie-type systems endowing with transformation Lie-groups $S L(2, \mathbb{R})$ can be obtained. The solution of second-order linear homogeneous differential equation is an integrating factor of the corresponding Riccati differential equation. The numerical schemes which are developed to fulfil the Lie-group property have better accuracy and stability than other schemes. We demonstrate that upon applying the group-preserving scheme (GPS) to the logistic differential equation, it is not only qualitatively correct for all values of time stepsize $h$, and is also the most accurate one among all numerical schemes compared in this paper. The group-preserving schemes derived for the Riccati differential equation, second-order linear homogeneous and non-homogeneous differential equations, the Abel differential equation and higher-order nonlinear differential equations all have accuracy better than $O\left(h^{2}\right)$.
\end{abstract}

Keywords: group-preserving scheme, Riccati equation, Abel equation, Logistic equation, linear fraction map

\section{Introduction}

The Lie-groups play an important role to understand the geometric structure of differential equations, which are very useful in the development of some superior numerical methods to integrate ordinary differential equations (ODEs), and to retain the invariant property of dynamical system. By sharing the geometric structure and invariant with the original ODEs, the new methods are more accurate, more stable and more effective than conventional numerical methods. In the last few decades there has been a substantial development in the geometric integrators to solve ODEs evolving on the Lie groups (Munthe-Kass, 1999; Iserles et al., 2000; Liu, 2007; Hochbruck \& Ostermann, 2010). The Lie-group schemes find numerical approximations to the solution of

$$
\dot{\mathbf{Y}}=\mathbf{A}(\mathbf{Y}, t) \mathbf{Y}, \quad \mathbf{Y}(0)=\mathbf{Y}_{0},
$$

where the exact solution $\mathbf{Y}$ is a matrix Lie group with $\mathbf{A}$ being a matrix function on the associated Lie algebra.

In this paper we introduce the integrating factor methods for the Riccati, Abel and higher-order nonlinear differential equations, and then use a Lie-group $S L(2, \mathbb{R})$ approach for the solutions of the above equations. The Lie-group $S L(2, \mathbb{R})$ has been used to develop the shooting method to solve the generalized Sturm-Liouville problem by Liu (2012), to solve a singular $\phi$-Laplacian nonlinear ODE by Liu (2013a), and to solve a nonlinear heat transfer equation by Hashemi (2015).

For the first-order linear ODE:

$$
\dot{y}(t)+p(t) y(t)=q(t),
$$

we have already learned that the integrating factor is defined by

$$
I(t)=\exp \left[\int_{0}^{t} p(\xi) d \xi\right],
$$


which can transform Equation (1), after multiplying both the sides by $I(t)$, into a total differential form on the left-hand side:

$$
\frac{d}{d t}[I(t) y(t)]=I(t) q(t)
$$

Such that the general solution of Equation (1) can be obtained simply by integrating the above equation.

The key point by passing from Equation (1) to Equation (3) is the idea of integrating factor, leading to a total differential form in Equation (3). This idea can be extended to the Riccati equation. It not only provides a total differential form of the left-hand side and also linearizes the nonlinear differential equation exactly, of which the integrating factor $u(t)$ is a solution of a second-order linear ODE:

$$
\ddot{u}(t)+a(t) \dot{u}(t)+b(t) u=0 .
$$

In the course of Engineering Mathematics we may teach the students to write Equation (4) as a system of two first-order ODEs by

$$
\frac{d}{d t}\left[\begin{array}{l}
\dot{u}(t) \\
u(t)
\end{array}\right]=\left[\begin{array}{cc}
-a(t) & -b(t) \\
1 & 0
\end{array}\right]\left[\begin{array}{l}
\dot{u}(t) \\
u(t)
\end{array}\right] .
$$

However, letting

$$
p(t)=\exp \left[-\int_{0}^{t} a(\xi) d \xi\right]
$$

be the integrating factor of Equation (4) we have

$$
\frac{d}{d t}\left(\frac{\dot{u}(t)}{p(t)}\right)=-\frac{b(t) u}{p(t)}
$$

It shows again that the left-hand side can be managed to a total differential form by using the concept of integrating factor. Furthermore, from Equation (7) we have a self-adjoint system:

$$
\frac{d}{d t}\left[\begin{array}{c}
\dot{u}(t) / p(t) \\
u(t)
\end{array}\right]=\left[\begin{array}{cc}
0 & -b(t) / p(t) \\
p(t) & 0
\end{array}\right]\left[\begin{array}{c}
\dot{u}(t) / p(t) \\
u(t)
\end{array}\right] .
$$

This system is better than system (5), because it allows an internal symmetry of $S L(2, \mathbb{R})$.

Although, the author (Liu, 2001; Liu, 2013b; Liu, 2015) has previously developed several general Lie-group theories for the general nonlinear dynamical system, the specialization to the Lie-group $S L(2, \mathbb{R})$ for the secondorder linear ODE and Riccati equation and finding that the solution of the former is an integrating factor of the latter is interesting, which deserves a further attention by sharing the Lie-group property to develop more efficient numerical scheme for these two equations.

There are many ODEs that the concept of integrating factor can be used to derive new forms of ODEs, of which the internal symmetry appears naturally. Liu (2001) was the first by using the technique of integrating factor to transform the nonlinear ODEs into an augmented system in the Minkowski space, where the length of state vector plays the role of integrating factor. Then, Liu (2006) introduced the concept of multiple integrating factors to solve the ODEs with multiple constraints.

Many numerical methods have been developed for the general purpose of integrating the system of ODEs to obtain numerical solutions. Among those numerical methods, the most known are the Euler method and the Runge-Kutta method, but less known is the group-preserving scheme (Liu, 2001). These methods discretize the differential equations system to produce difference equations or mappings. The different methods obtain different mappings from the same differential equations, but they have the same aim in that the dynamics of the mapping should correspond as closely as possible to the dynamics of original differential equations. However, it is a major question whether the corresponding approximation has the same behavior as the original differential equations. In recent years, a major area is to study the difference in the exact dynamics of differential equations and the dynamics of the corresponding discretized mappings. It is found that the most serious problems for the numerical discretizations are the appearance of so-called spurious solutions and ghost fixed points in the discretized dynamics. For example, Chen and Solis (1998) have investigated the appearance of spurious solutions when some first-order ODEs are discretized by using the Runge-Kutta methods, and showed that the resulting schemes may produce unrelated bifurcation phenomena. 
Of all the first-order nonlinear differential equations, the Riccati differential equation occupies perhaps the most important place, because it is closely related to the second-order linear homogeneous differential equations, which appear in many physical applications. In the Engineering Mathematics we have learned some special functions and series solutions for the Bessel equation, the Legendre equation, the Airy equation, etc., but from the computational point of view it is not convenient to use these methods to calculate the solutions. In this paper we attempt to develop suitable numerical schemes for the Riccati differential equation, second-order linear differential equations, and the Abel differential equation. Although the above systems will be shown exhibiting certain symmetry, there has no development of the numerical scheme to preserve it. Really, there have many issues concerning the improvement of the quality of solution by producing numerical solutions of ODEs that possess symmetries and structures and also utilize the symmetries to ensure the computational stability.

The arrangements of this paper are given as follows. In Section 2 we introduce a Lie-group symmetry for the Riccati differential equation, and then we explain that its integrating factor is a solution of the second-order linear differential equation in Section 3. Based on the Lie-symmetry we develop a group-preserving scheme for the Riccati differential equation in Section 4, and the group-preserving scheme for the logistic differential equation is developed in Section 5, which is compared with other numerical schemes. The group-preserving scheme for secondorder linear differential equation is developed in Section 6, while that for second-order linear non-homogeneous differential equation is developed in Section 7. The group-preserving schemes for the Abel differential equation and other higher-order nonlinear differential equations are developed in Section 8. Finally, the conclusions are drawn in Section 9.

\section{Symmetry Group}

The following Riccati differential equation:

$$
\frac{d y}{d t}=r(t)-2 q(t) y-p(t) y^{2}
$$

can be written as

$$
\frac{d y}{d t}+[p(t) y+q(t)] y=r(t)-q(t) y .
$$

Upon defining the integrating factor:

$$
u(t):=u(0) \exp \left[\int_{0}^{t}[p(\xi) y(\xi)+q(\xi)] d \xi\right],
$$

Equation (10) becomes

$$
\frac{d}{d t}(u y)=r(t) u-q(t) u y .
$$

On the other hand, from Equation (11) it follows that

$$
\frac{d u}{d t}=p(t) u y+q(t) u
$$

Equations (12) and (13) together constitute a linear system:

$$
\frac{d}{d t}\left[\begin{array}{c}
u y \\
u
\end{array}\right]=\mathbf{A}\left[\begin{array}{c}
u y \\
u
\end{array}\right],
$$

where

$$
\mathbf{A}:=\left[\begin{array}{cc}
-q(t) & r(t) \\
p(t) & q(t)
\end{array}\right]
$$

We thus represent the Riccati differential equation in the projective space $(u y, u)$ as a system of two first-order linear ODEs.

The above coefficient matrix A satisfying tr $\mathbf{A}=0$ is a Lie algebra of $S L(2, \mathbb{R})$, where tr denotes the trace. Thus, the one-parameter subgroup generated by $\mathbf{A}$ gives the following transformation formula for $(u y, u)$ :

$$
\left[\begin{array}{c}
u(t) y(t) \\
u(t)
\end{array}\right]=\mathbf{G}(t)\left[\begin{array}{c}
u(0) y(0) \\
u(0)
\end{array}\right]
$$


where $\mathbf{G}$ is an element of the two-dimensional special linear group, satisfying

$$
\operatorname{det} \mathbf{G}(t)=1, \mathbf{G}(0)=\mathbf{I}_{2},
$$

in which det stands for the determinant, and $\mathbf{I}_{2}$ is a second order identity matrix. The above fact prompts us to develop a suitable numerical scheme to preserve the Lie-group property.

\section{The Second-order Linear Differential Equations}

It follows from Equations (13) and (12) a second-order linear ODE for $u$ :

$$
\ddot{u}+a(t) \dot{u}+b(t) u=0,
$$

where

$$
\begin{aligned}
& a(t)=\frac{-\dot{p}(t)}{p(t)} \\
& b(t)=\left[\frac{\dot{p}(t)}{p(t)}-q(t)\right] q(t)-p(t) r(t)-\dot{q}(t) .
\end{aligned}
$$

For a given $a(t)$ it follows directly that

$$
p(t)=\exp \left[-\int_{0}^{t} a(\xi) d \xi\right]
$$

On the other hand, without lost any generality we can simply take $q(t)=0$, such that $b(t)=-p(t) r(t)$ holds.

If $\mathbf{G}$ is a fundamental matrix of Equation (14) satisfying

$$
\dot{\mathbf{G}}=\mathbf{A G}, \quad \mathbf{G}(0)=\mathbf{I}_{2},
$$

then from Equation (16), with its $u y$ replaced by $\dot{u} / p$ due to $q(t)=0$ in Equation (13), we can obtain

$$
\left[\begin{array}{c}
\dot{u}(t) / p(t) \\
u(t)
\end{array}\right]=\left[\begin{array}{cc}
G_{11}(t) & G_{12}(t) \\
G_{21}(t) & G_{22}(t)
\end{array}\right]\left[\begin{array}{c}
\dot{u}(0) / p(0) \\
u(0)
\end{array}\right] .
$$

Noting that $G_{21}(t)$ and $G_{22}(t)$ are linearly independent solutions of Equation (18), because in Equation (23),

$$
u(t)=\frac{\dot{u}(0)}{p(0)} G_{21}(t)+u(0) G_{22}(t)
$$

is a general solution of Equation (18).

Differentiating the second equation with respect to $t$ and comparing it with the first equation, we obtain

$$
\frac{\dot{G}_{21}(t)}{p(t)}=G_{11}(t), \frac{\dot{G}_{22}(t)}{p(t)}=G_{12}(t) .
$$

Substituting the above two equations for $G_{11}(t)$ and $G_{12}(t)$ into the identity $G_{11}(t) G_{22}(t)-G_{21}(t) G_{12}(t)=1$, we can derive

$$
\left|\begin{array}{ll}
G_{22}(t) & G_{21}(t) \\
\dot{G}_{22}(t) & \dot{G}_{21}(t)
\end{array}\right|=p(t)=\exp \left[-\int_{0}^{t} a(\xi) d \xi\right],
$$

which is known as the Abel identity. It is clear that such an identity is a direct result of the Lie-group property (17).

\section{Numerical Scheme for Riccati Differential Equation}

A numerical scheme based on the Lie-group property can be utilized to enhance the computational accuracy and efficiency. The time-centered Euler scheme for Equation (14) is

$$
\left[\begin{array}{c}
u_{n+1} y_{n+1} \\
u_{n+1}
\end{array}\right]=\mathbf{G}(\bar{n})\left[\begin{array}{c}
u_{n} y_{n} \\
u_{n}
\end{array}\right],
$$

where

$$
\mathbf{G}(\bar{n}):=[\mathbf{I}-\tau \mathbf{A}(\bar{n})]^{-1}[\mathbf{I}+\tau \mathbf{A}(\bar{n})] .
$$


Here $n \in \mathbb{Z}^{+}, u_{n}$ and $y_{n}$ denote respectively the value of $u$ and $y$ at the discrete time $t_{n}$, and $\tau$ is one half of the stepsize $h$, that is, $\tau:=h / 2=\Delta t / 2$. The notation $\bar{n}$ is used to denote $n+1 / 2$, and $\mathbf{A}(\bar{n})$ means that $\mathbf{A}$ takes value at $t_{n}+\tau$. From Equation (15) we have

$$
\mathbf{A}(\bar{n})=\left[\begin{array}{cc}
-q(\bar{n}) & r(\bar{n}) \\
p(\bar{n}) & q(\bar{n})
\end{array}\right] .
$$

Substituting the above $\mathbf{A}(\bar{n})$ into Equation (27) and through some manipulations we find that

$$
\mathbf{G}(\bar{n})=\frac{1}{1-\tau^{2}\left[p(\bar{n}) r(\bar{n})+q^{2}(\bar{n})\right]}\left[\begin{array}{cc}
{[1-\tau q(\bar{n})]^{2}+\tau^{2} p(\bar{n}) r(\bar{n})} & h r(\bar{n}) \\
h p(\bar{n}) & {[1+\tau q(\bar{n})]^{2}+\tau^{2} p(\bar{n}) r(\bar{n})}
\end{array}\right] .
$$

A straightforward calculation shows that $\operatorname{det} \mathbf{G}(\bar{n})=1$ holds. Thus the above numerical scheme meets the requirement for preserving the Lie-group property. Substituting Equation (29) for $\mathbf{G}(\bar{n})$ into Equation (26) and taking the projection, we obtain

$$
y_{n+1}=\frac{\tau^{2} p(\bar{n}) r(\bar{n}) y_{n}+[1-\tau q(\bar{n})]^{2} y_{n}+h r(\bar{n})}{h p(\bar{n}) y_{n}+\tau^{2} p(\bar{n}) r(\bar{n})+[1+\tau q(\bar{n})]^{2}},
$$

which is a group-preserving scheme (GPS) for the Riccati differential equation (9).

For the purpose of comparison we give a numerical example to calculate the solution of Equation (9) with $p(t)=$ $-2 \cos t, q(t)=1 / 2+2 t \cos t$, and $r(t)=1+t+2 t^{2} \cos t$, of which the exact solution is $y(t)=t-[\sin t-\cos t+c \exp t]^{-1}$, where $c=1-1 / y(0)$. Starting from the initial condition $y(0)=-1$, using $h=0.001$ and substituting the above coefficient functions into Equation (30) we can obtain the numerical solution, which upon comparing with the closed-form solution we show the solution error, which is obtained by substracting the numerical solution from the closed-form solution, in Figure 1. It can be seen that the accuracy of scheme (30) is better than the order of $O\left(h^{2}\right)$.

From Equation (30) we can conclude that a suitable scheme for the Riccati differential equation is a linear fraction map of the following form:

$$
y_{n+1}=\frac{A y_{n}+B}{C y_{n}+D}
$$

with

$$
\left[\begin{array}{cc}
A & B \\
C & D
\end{array}\right] \in S L(2, \mathbb{R}),\left.\quad\left[\begin{array}{cc}
A & B \\
C & D
\end{array}\right]\right|_{h=0}=\mathbf{I}_{2}
$$

\section{Numerical Schemes for Logistic Differential Equation}

Now, let us apply scheme (30) to the logistic differential equation:

$$
\dot{y}=\lambda y(1-y)
$$

whose solution is given by

$$
y(t)=\frac{y_{0}}{\exp [-\lambda t]+(1-\exp [-\lambda t]) y_{0}}
$$

with $y(0)=y_{0}$. Inserting $p=\lambda, q=-\lambda / 2$ and $r=0$ into Equation (30), we obtain a discretized map for the logistic differential equation:

$$
y_{n+1}=\frac{[4+\lambda h]^{2} y_{n}}{16 \lambda h y_{n}+[4-\lambda h]^{2}}=: g\left(y_{n}, \lambda h\right) .
$$

Obviously, $g$ has only two fixed points $y=0$ and $y=1$, and because of

$$
\begin{aligned}
& g^{\prime}(0)=\frac{[4+\lambda h]^{2}}{[4-\lambda h]^{2}}>1, \quad \forall \lambda>0, \\
& g^{\prime}(1)=\frac{[4-\lambda h]^{2}}{[4+\lambda h]^{2}}<1, \quad \forall \lambda>0,
\end{aligned}
$$

the first fixed point is unstable and the second one is stable when $\lambda>0$. Conversely,

$$
\begin{aligned}
& g^{\prime}(0)<1, \quad \forall \lambda<0, \\
& g^{\prime}(1)>1, \quad \forall \lambda>0
\end{aligned}
$$


lead to the fixed point $y=0$ to be stable and the fixed point $y=1$ to be unstable when $\lambda<0$. Thus, the map (35) has the same properties as the original equation (33), no matter what $\lambda$ is. Furthermore, by the induction method the solution of Equation (35) is found to be

$$
y_{n}=\frac{y_{0}}{\left(\frac{4-\lambda h}{4+\lambda h}\right)^{2 n}+\left(1-\left[\frac{4-\lambda h}{4+\lambda h}\right]^{2 n}\right) y_{0}} .
$$

It has the same form as Equation (34), and no matter what the initial value $y_{0}$ is and what $\lambda$ is, it tends to the stable fixed point.

In Figure 2, where $\lambda=2, h=0.001$ and $y_{0}=0.1$ were used, we compare the numerical solution with the closedform solution in terms of solution error. The accuracy of scheme (35) can be seen almost in the order of $O\left(h^{3}\right)$. Also the comparisons with the following schemes are made in Figure 2:

$$
\begin{aligned}
& y_{n+1}=\frac{y_{n}}{\lambda h y_{n}+1-\lambda h}, \\
& y_{n+1}=\frac{(1+\lambda h) y_{n}}{\lambda h y_{n}+1} \text {, } \\
& y_{n+1}=\frac{(2+\lambda h) y_{n}}{2 \lambda h y_{n}+2-\lambda h} \text {, } \\
& y_{n+1}=\frac{[1-\exp (-\lambda h)] y_{n}+1+2[1-\exp (-\lambda h)]}{2[1-\exp (-\lambda h)] y_{n}+1+[1-\exp (-\lambda h)]} y_{n} .
\end{aligned}
$$

Here, the first two schemes are proposed by Wang et al. (1992), and the third is the combination of the first two schemes (Gander \& Meyer-Spasche, 2000, p.224). The fourth is called by Mickens (1999) the nonstandard finite-difference scheme, the factor $1-\exp (-\lambda h)$ in which is known as the denominator function.

Th accuracies of the above numerical schemes are compared in Figure 2, which indicate that scheme (39) has accuracy better than the order of $O\left(h^{2}\right)$, the accuracy of shemes (37) and (38) is better than the order of $O(h)$, but scheme (40) has accuracy in the order of $O(h)$. As shown in Figure 2, schemes (37) and (38) lead to the same absolute solution error but with difference in sign, and so we introduce a scheme being the average of them:

$$
y_{n+1}=\frac{y_{n}}{2-2 \lambda h\left(1-y_{n}\right)}+\frac{(1+\lambda h) y_{n}}{2+2 \lambda h y_{n}},
$$

whose accuracy is better than schemes (37) and (38), but is worse than scheme (39).

Now we discuss something about the group-preserving scheme. When $\lambda h<1$, scheme (37) can be written as the form of Equation (31) with

$$
\left[\begin{array}{ll}
A & B \\
C & D
\end{array}\right]=\left[\begin{array}{cc}
\frac{1}{\sqrt{1-\lambda h}} & 0 \\
\frac{\lambda h}{\sqrt{1-\lambda h}} & \frac{1-\lambda h}{\sqrt{1-\lambda h}}
\end{array}\right] .
$$

It satisfies condition (32), so that scheme (37) is a group-preserving scheme in the range $\lambda h<1$. However, in the range of $\lambda h>1$, scheme (37) fails to be a group-preserving scheme. Gander \& Meyer-Spasche (2000) have proved that scheme (37) behaves qualitatively correctly for $\lambda h<1$. For $1<\lambda h<2$, it converges to the correct limit oscillatorily. For $\lambda h>2$, it behaves wrongly. Thus, a scheme which can preserve the Lie-group property is very important. Scheme (38) is a group-preserving scheme if $\lambda h>-1$; otherwise, it fails to be a group-preserving scheme. Similarly, scheme (39) is a group-preserving scheme in the range $\lambda h<2$, and when $\lambda h>2$, it fails to be a group-preserving scheme. It is obvious that only the scheme (35) is a group-preserving for all $\lambda$. Schemes (40) and (41) are not of the linear fraction type as Equation (31) is, and thus are not of the group-preserving type.

There have several criteria to assess the performance of a numerical scheme, among, the order of accuracy, stability property and preserving the dynamics of the original ODEs are the most important three factors. In this regard, the above introduced group-preserving scheme (35) is the best one for the discretizations of the logistic differential equation (33).

\section{Numerical Scheme for Second-order Linear Differential Equations}


In order to find the numerical scheme for Equation (18), we let $\dot{u}=p(t) u y$ by Equation (13) due to $q(t)=0$; hence, from Equations (26) and (29) the numerical scheme for $u$ and $\dot{u}$ can be written as

$$
\left[\begin{array}{l}
u_{n+1} \\
\dot{u}_{n+1}
\end{array}\right]=\frac{1}{1+\tau^{2} b(\bar{n})}\left[\begin{array}{cc}
1-\tau^{2} b(\bar{n}) & \frac{h p(\bar{n})}{p(n)} \\
-\frac{h p(n+1) b(\bar{n})}{p(\bar{n})} & \frac{p(n+1)\left[1-\tau^{2} b(\bar{n})\right]}{p(n)}
\end{array}\right]\left[\begin{array}{l}
u_{n} \\
\dot{u}_{n}
\end{array}\right],
$$

where $p$ is defined in Equation (21). The above scheme is suitable to calculating the solution of the second-order linear differential equation (18), whose initial values of $u$ and $\dot{u}$ are specified.

Let us consider a special case

$$
\ddot{u}+b(t) u=0 .
$$

The general second-order linear homogeneous differential equations can always be transformed into the above type. For this case let $p=1$ in Equation (42) we obtain

$$
\left[\begin{array}{l}
u_{n+1} \\
\dot{u}_{n+1}
\end{array}\right]=\frac{1}{1+\tau^{2} b(\bar{n})}\left[\begin{array}{cc}
1-\tau^{2} b(\bar{n}) & h \\
-h b(\bar{n}) & 1-\tau^{2} b(\bar{n})
\end{array}\right]\left[\begin{array}{l}
u_{n} \\
\dot{u}_{n}
\end{array}\right] .
$$

Through some manipulations we obtain

$$
u_{n+1}=\frac{\left(2-\tau^{2}[b(\bar{n})+b(\bar{n}-1)]\right) u_{n}-\left[1+\tau^{2} b(\bar{n}-1)\right] u_{n-1}}{1+\tau^{2} b(\bar{n})} .
$$

It bears certain similarity with the following two schemes:

$$
u_{n+1}+u_{n-1}=2 \cos (h \sqrt{b(n)}) u_{n}
$$

developed by Mickens \& Ramadhani (1992), and

$$
\left[1+\frac{h^{2} b(n+1)}{12}\right] u_{n+1}+\left[1+\frac{h^{2} b(n-1)}{12}\right] u_{n-1}=2 \cos (h \sqrt{b(n)})\left[1+\frac{h^{2} b(n)}{12}\right] u_{n}
$$

generalized from the Mickens-Ramadhani scheme by Chen et al. (1993).

In order to compare the above three numerical schemes we let $b(t)=1 / t^{2}$ in the range $t \in[1,10]$. For such $b(t)$, Equation (43) has closed-form solution as follows:

$$
u(t)=\sqrt{t}\left[u(1) \cos \left(\frac{\sqrt{3}}{2} \ln t\right)+\frac{2 \dot{u}(1)-u(1)}{\sqrt{3}} \sin \left(\frac{\sqrt{3}}{2} \ln t\right)\right] .
$$

The solution error of the above three schemes are compared in Figure 3, where $u(1)=\dot{u}(1)=1$ were taken, and the stepsize $h=0.001$ was used. As denoted by Chen et al. (1993) the modified scheme (47) is of $O\left(h^{4}\right)$ and the Mickens-Ramadhani scheme (45) is of $O\left(h^{2}\right)$. It can be seen that the accuracy of scheme (45) is between schemes (46) and (47). However the latter two schemes do not share the Lie-group property when $b(t)$ is a time-varying function as this case is.

When we consider $b(t)=\omega^{2}$ for the linear harmonic oscillator equation,

$$
\ddot{u}+\omega^{2} u=0,
$$

the numerical scheme in Equation (44) is simplified to

$$
\left[\begin{array}{c}
u_{n+1} \\
\dot{u}_{n+1}
\end{array}\right]=\frac{1}{1+\tau^{2} \omega^{2}}\left[\begin{array}{cc}
1-\tau^{2} \omega^{2} & h \\
-h \omega^{2} & 1-\tau^{2} \omega^{2}
\end{array}\right]\left[\begin{array}{l}
u_{n} \\
\dot{u}_{n}
\end{array}\right] .
$$

Here we require $\tau^{2} \omega^{2}<1$. It deserves to note that the above scheme conserves the total energy $E:=\left[\dot{u}^{2}+(\omega u)^{2}\right] / 2$, that is,

$$
\left[\omega u_{n+1}\right]^{2}+\dot{u}_{n+1}^{2}=\left[\omega u_{n}\right]^{2}+\dot{u}_{n}^{2}=2 E .
$$


On the other hand, when applying the nonstand difference scheme of Mickens to Equation (49) gives

$$
\left[\begin{array}{c}
u_{n+1} \\
\dot{u}_{n+1}
\end{array}\right]=\left[\begin{array}{cc}
\cos (h \omega) & \frac{1}{\omega} \sin (h \omega) \\
-\omega \sin (h \omega) & \cos (h \omega)
\end{array}\right]\left[\begin{array}{c}
u_{n} \\
\dot{u}_{n}
\end{array}\right] .
$$

Refer Equations (2.40a) and (2.40b) in (Mickens, 2000). Obviously, Equation (51) also holds for this scheme. Comparing the coefficients matrices in schemes (50) and (52), we note that both the transition matrices are of $S L(2, \mathbb{R})$, and

$$
\begin{aligned}
& \frac{1-\tau^{2} \omega^{2}}{1+\tau^{2} \omega^{2}}=1-2 \tau^{2} \omega^{2}+2\left(\tau^{2} \omega^{2}\right)^{2}+\cdots \\
& \cos (h \omega)=1-2 \tau^{2} \omega^{2}+\frac{2\left(\tau^{2} \omega^{2}\right)^{2}}{3}+\cdots
\end{aligned}
$$

make the above two schemes slightly different in the order of $O\left(h^{4}\right)$.

\section{Numerical Scheme for Second-order Linear Non-homogeneous Differential Equations}

Now, let us consider the following second-order linear non-homogeneous differential equation:

$$
\ddot{u}+a(t) \dot{u}+b(t) u=f(t)
$$

with a forcing term $f(t)$. It is equivalent to solve the following non-homogeneous linear system:

$$
\frac{d}{d t}\left[\begin{array}{c}
\dot{u}(t) / p(t) \\
u(t)
\end{array}\right]=\left[\begin{array}{cc}
0 & -b(t) / p(t) \\
p(t) & 0
\end{array}\right]\left[\begin{array}{c}
\dot{u}(t) / p(t) \\
u(t)
\end{array}\right]+\left[\begin{array}{c}
f(t) / p(t) \\
0
\end{array}\right] .
$$

The solution is given by

$$
\begin{aligned}
u(t) & =\left[G_{22}(t) G_{11}\left(t_{i}\right)-G_{21}(t) G_{12}\left(t_{i}\right)\right] u\left(t_{i}\right)+\left[G_{21}(t) G_{22}\left(t_{i}\right)-G_{22}(t) G_{21}\left(t_{i}\right)\right] \frac{\dot{u}\left(t_{i}\right)}{p\left(t_{i}\right)} \\
& -G_{22}(t) \int_{t_{i}}^{t} \frac{G_{21}(\eta) f(\eta)}{p(\eta)} d \eta+G_{21}(t) \int_{t_{i}}^{t} \frac{G_{22}(\eta) f(\eta)}{p(\eta)} d \eta, \\
\frac{\dot{u}(t)}{p(t)} & =\left[G_{12}(t) G_{11}\left(t_{i}\right)-G_{11}(t) G_{12}\left(t_{i}\right)\right] u\left(t_{i}\right)+\left[G_{11}(t) G_{22}\left(t_{i}\right)-G_{12}(t) G_{21}\left(t_{i}\right)\right] \frac{\dot{u}\left(t_{i}\right)}{p\left(t_{i}\right)} \\
& -G_{12}(t) \int_{t_{i}}^{t} \frac{G_{21}(\eta) f(\eta)}{p(\eta)} d \eta+G_{11}(t) \int_{t_{i}}^{t} \frac{G_{22}(\eta) f(\eta)}{p(\eta)} d \eta,
\end{aligned}
$$

where $u\left(t_{i}\right)$ and $\dot{u}\left(t_{i}\right)$ are the initial values prescribed at time $t_{i}$. The second line in Equation (55) is really a particular solution of Equation (53). It leads to the same result as that obtained by the method of variation of parameters. In Equation (54) the first equation

$$
\frac{d}{d t}\left[\frac{1}{p(t)} \frac{d u}{d t}\right]+\frac{b(t)}{p(t)} u=\frac{f(t)}{p(t)}
$$

is known as the non-homogeneous Sturm-Liouville equation, and the differential operator

$$
\frac{d}{d t}\left[\frac{1}{p(t)} \frac{d}{d t}\right]+\frac{b(t)}{p(t)}
$$

is self-adjoint. The usual way to systemize Equation (53) is given by

$$
\frac{d}{d t}\left[\begin{array}{l}
\dot{u}(t) \\
u(t)
\end{array}\right]=\left[\begin{array}{cc}
-a(t) & -b(t) \\
1 & 0
\end{array}\right]\left[\begin{array}{l}
\dot{u}(t) \\
u(t)
\end{array}\right]+\left[\begin{array}{c}
f(t) \\
0
\end{array}\right]
$$

However, the system matrix of this equation does not has any special structure in it. Thus, the numerical method based on this equation will not capture the main Lie-group property inherent in such an equation. 
For the non-homogeneous equation (53) we can let $t \rightarrow t_{n+1}, t_{i} \rightarrow t_{n}$, and apply the trapezoidal quadrature to the integrals in Equations (55) and (56) to obtain

$$
\begin{aligned}
u_{n+1} & =\frac{G_{21}(\bar{n}) \dot{u}_{n}}{p(n)}+G_{22}(\bar{n}) u_{n}+\frac{\tau G_{21}(\bar{n}) f(n)}{p(n)}, \\
\frac{\dot{u}_{n+1}}{p(n+1)} & =\frac{G_{11}(\bar{n}) \dot{u}_{n}}{p(n)}+G_{12}(\bar{n}) u_{n}+\frac{\tau G_{11}(\bar{n}) f(n)}{p(n)}+\frac{\tau f(n+1)}{p(n+1)},
\end{aligned}
$$

where

$$
\mathbf{G}(\bar{n})=\frac{1}{1+\tau^{2} b(\bar{n})}\left[\begin{array}{cc}
1-\tau^{2} b(\bar{n}) & \frac{-h b(\bar{n})}{p(\bar{n})} \\
h p(\bar{n}) & 1-\tau^{2} b(\bar{n})
\end{array}\right] .
$$

In order to investigate the accuracy of the above numerical scheme we consider the following differential equation:

$$
t^{2} \ddot{u}+u=\ln t
$$

in the range $t \in[1,10]$, with $u(1)=2$ and $\dot{u}(1)=1$. Equation (60) has a closed-form solution given as follows:

$$
u(t)=\sqrt{t}\left[u(1) \cos \left(\frac{\sqrt{3}}{2} \ln t\right)+\frac{2 \dot{u}(1)-u(1)}{\sqrt{3}} \sin \left(\frac{\sqrt{3}}{2} \ln t\right)\right]+1+\ln t,
$$

where

$$
C_{1}=u(1)-1=1, \quad C_{2}=\frac{2 \dot{u}(1)-u(1)-1}{\sqrt{3}}=\frac{-1}{\sqrt{3}} .
$$

Substituting $p(t)=1, b(t)=1 / t^{2}$ and $f(t)=\ln t / t^{2}$ into the above scheme we can calculate $u$, whose error is shown in Figure 4, where the stepsize $h=0.001$ was used in the calculation. It can be seen that the accuracy is better than the order of $O\left(h^{2}\right)$.

\section{Abel Differential Equation and Higher-order Nonlinear ODE}

\subsection{The Integrating Factor for Abel Differential Equation}

A direct extension of the Riccati differential equation is the Abel differential equation (Sachdev, 1991):

$$
\frac{d y}{d t}=r(t)-2 q(t) y-p(t) y^{2}-e(t) y^{3}, y(0)=y_{0},
$$

which can be written as

$$
\frac{d y}{d t}+\left[q(t)+p(t) y+e(t) y^{2}\right] y=r(t)-q(t) y
$$

Upon defining the integrating factor:

$$
u(t):=u(0) \exp \left[\int_{0}^{t}\left[q(\xi)+p(\xi) y(\xi)+e(\xi) y^{2}(\xi)\right] d \xi\right],
$$

Equation (62) becomes

$$
\frac{d}{d t}(u y)=r(t) u-q(t) u y .
$$

On the other hand, from Equation (64) it follows that

$$
\frac{d u}{d t}=q(t) u+p(t) u y+e(t) u y^{2} .
$$

Equations (65) and (66) together can be written as

$$
\frac{d}{d t}\left[\begin{array}{c}
u y \\
u
\end{array}\right]=\mathbf{A}\left[\begin{array}{c}
u y \\
u
\end{array}\right],
$$

where

$$
\mathbf{A}:=\left[\begin{array}{cc}
-q(t) & r(t) \\
p(t)+e(t) y & q(t)
\end{array}\right] .
$$


Because the coefficient matrix A satisfies $\operatorname{tr} \mathbf{A}=0$, the resulting Lie-group is $S L(2, \mathbb{R})$, which is a representation of the Abel differential equation in the projective space $(u y, u)$ as a system of two first-order differential equations. However, the above $\mathbf{A}$ is different from that in Equation (15) with an extra term $e(t) y=e(t) u y / u$, which renders Equation (67) a quasi-linear system endowing with a local Lie-algebra element of $\operatorname{sl}(2, \mathbb{R})$.

\subsection{The $S L(2, \mathbb{R})$ Group-preserving Scheme}

According to Equation (67), we can develop an implicit scheme based on $S L(2, \mathbb{R})$ for the integration of Equation (62), of which one can view

$$
\overline{\mathbf{A}}_{k}:=\left[\begin{array}{cc}
-\bar{q}_{k} & \bar{r}_{k} \\
\bar{p}_{k}+\bar{e}_{k} \bar{y}_{k} & \bar{q}_{k}
\end{array}\right]
$$

as a constant matrix within a small time increment, where $\bar{t}_{k}=(1-\theta) t_{k}+\theta t_{k+1}, \bar{q}_{k}=q\left(\bar{t}_{k}\right), \bar{r}_{k}=r\left(\bar{t}_{k}\right), \bar{p}_{k}=p\left(\bar{t}_{k}\right)$, $\bar{e}_{k}=e\left(\bar{t}_{k}\right)$ and $\bar{y}_{k}=(1-\theta) y_{k}+\theta y_{k+1}$. The corresponding Lie-group $\mathbf{G}_{k} \in S L(2, \mathbb{R})$ is obtained by using the exponential mapping:

$$
\mathbf{G}_{k}=\exp \left(h \overline{\mathbf{A}}_{k}\right)
$$

Let

$$
a_{k}:=\bar{p}_{k}+\bar{e}_{k} \bar{y}_{k}
$$

For the special case with $r(t)=0$ we have

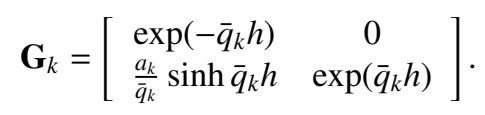

For the case $r(t) \neq 0$, through some derivations we can obtain

$$
\begin{aligned}
\mathbf{G}_{k} & =\left[\begin{array}{cc}
\cosh \omega_{k} h-\frac{\bar{q}_{k}}{\omega_{k}} \sinh \omega_{k} h & \frac{\bar{r}_{k}}{\omega_{k}} \sinh \omega_{k} h \\
\frac{\omega_{k}^{2}-\bar{q}_{k}^{2}}{\omega_{k} \bar{r}_{k}} \sinh \omega_{k} h & \cosh \omega_{k} h+\frac{\bar{q}_{k}}{\omega_{k}} \sinh \omega_{k} h
\end{array}\right], \text { if } \bar{q}_{k}^{2}+a_{k} \bar{r}_{k}>0, \\
\mathbf{G}_{k} & =\left[\begin{array}{cc}
\cos \omega_{k} h-\frac{\bar{q}_{k}}{\omega_{k}} \sin \omega_{k} h & \frac{\bar{r}_{k}}{\omega_{k}} \sin \omega_{k} h \\
-\frac{\omega_{k}^{2}+\bar{q}_{k}^{2}}{\omega_{k} \bar{r}_{k}} \sin \omega_{k} h & \cos \omega_{k} h+\frac{\bar{q}_{k}}{\omega_{k}} \sin \omega_{k} h
\end{array}\right], \text { if } \bar{q}_{k}^{2}+a_{k} \bar{r}_{k}<0 .
\end{aligned}
$$

In above, $\omega_{k}=\sqrt{\left|\bar{q}_{k}^{2}+a_{k} \bar{r}_{k}\right|}$.

This scheme is implicit because it also depends on $y_{k+1}$, which requires an iteration to determine the value of $\left(z_{k+1}:=u_{k+1} y_{k+1}, u_{k+1}\right)$ at the next time step. The numerical process of group-preserving scheme (GPS) is given as follows.

(i) Give $0 \leq \theta \leq 1$, for example $\theta=1 / 2$.

(ii) Give an initial $\left(z_{0}, u_{0}\right)=\left(u_{0} y_{0}, u_{0}\right)$ at an initial time $t=t_{0}$ and a time stepsize $h$.

(iii) For $k=0,1, \ldots$, we repeat the following computations to a specified terminal time $t=t_{f}$. We first apply the forward Euler method to Equation (67):

$$
\begin{aligned}
z_{k+1} & =z_{k}-h q\left(t_{k}\right) z_{k}+h r\left(t_{k}\right) u_{k}, \\
u_{k+1} & =u_{k}+\left[p\left(t_{k}\right)+e\left(t_{k}\right) y_{k}\right] z_{k}+h q\left(t_{k}\right) u_{k}, \\
y_{k+1} & =\frac{z_{k+1}}{u_{k+1}}
\end{aligned}
$$

With the above $\left(z_{k+1}, u_{k+1}\right)$ generated from an Euler step as an initial guess we iteratively solve the new $\left(z_{k+1}, u_{k+1}\right)$ by

$$
\begin{aligned}
& \bar{y}_{k}=(1-\theta) y_{k}+\theta y_{k+1}, \\
& {\left[\begin{array}{l}
\hat{z}_{k+1} \\
\hat{y}_{k+1}
\end{array}\right]=\mathbf{G}_{k}\left[\begin{array}{l}
y_{k} \\
z_{k}
\end{array}\right],} \\
& y_{k+1}=\frac{\hat{z}_{k+1}}{\hat{u}_{k+1}} .
\end{aligned}
$$


If $\left(\hat{z}_{k+1}, \hat{u}_{k+1}\right)$ converges according to a given stopping criterion, such that,

$$
\sqrt{\left(\hat{z}_{k+1}-z_{k+1}\right)^{2}+\left(\hat{u}_{k+1}-u_{k+1}\right)^{2}}<\varepsilon
$$

then go to (iii) for the next time step; otherwise, let $z_{k+1}=\hat{z}_{k+1}$ and $u_{k+1}=\hat{u}_{k+1}$ and go to Equation (75).

For the Abel differential equation, there are only rare cases that the analytical solutions exist as summarized in Murphy (1960). In general the solutions are implicit. For example,

$$
\frac{d y}{d t}=a_{0} y+b_{0} y^{3}, y(0)=y_{0}
$$

has an analytical solution:

$$
y^{2}(t)\left(C e^{-2 a_{0} t}-b_{0}\right)=a_{0},
$$

where $C=a_{0} / y_{0}^{2}+b_{0}$.

We fix $a_{0}=2, b_{0}=1$ and $y_{0}=1$, and compare the solutions obtained by the GPS with the analytical solutions in Table 1 .

Table 1. Comparing exact and GPS solutions for an Abel equation

\begin{tabular}{l|l|l|l}
\hline$t$ & GPS & Exact & Absolute error \\
\hline 0.01 & 1.0307722 & 1.0307722 & $8.388 \times 10^{-9}$ \\
\hline 0.02 & 1.0631835 & 1.0631835 & $1.884 \times 10^{-8}$ \\
\hline 0.03 & 1.0973910 & 1.0973910 & $3.188 \times 10^{-8}$ \\
\hline 0.04 & 1.1335744 & 1.1335744 & $4.817 \times 10^{-8}$ \\
\hline 0.05 & 1.1719408 & 1.1719407 & $6.859 \times 10^{-8}$ \\
\hline 0.06 & 1.2127301 & 1.2127300 & $9.429 \times 10^{-8}$ \\
\hline 0.07 & 1.2562224 & 1.2562223 & $1.268 \times 10^{-7}$ \\
\hline 0.08 & 1.3027468 & 1.3027466 & $1.681 \times 10^{-7}$ \\
\hline 0.09 & 1.3526930 & 1.3526927 & $2.211 \times 10^{-7}$ \\
\hline 0.1 & 1.4065270 & 1.4065267 & $2.894 \times 10^{-7}$ \\
\hline
\end{tabular}

In order to test the accuracy of the above GPS we let $y=\sin t$ be an exact solution. Substituting $p(t)=0.5$, $q(t)=1 / 2, e(t)=-1, y=\sin t$ and $\dot{y}=\cos t$ into Equation (62) we can obtain $r(t)$. Under $h=0.001$ and $\varepsilon=10^{-10}$ we use the above GPS to calculate $y$, whose number of iterations and the absolute error of numerical solution are shown in Figure 5. Although under a stringent convergence criterion $\varepsilon=10^{-10}$, the number of iterations is either three or four. It can be seen that the accuracy is better than the order of $O\left(h^{2}\right)$.

Let us consider (Adeyeye et al., 2015)

$$
\frac{d y}{d t}=1-y+y^{3}, y(0)=0
$$

which is a special case of Equation (62) with $p(t)=0, q(t)=1 / 2, e(t)=-1$, and $r(t)=1$. Under $h=0.001$ and $\varepsilon=10^{-10}$ we use the GPS to calculate $y$, whose number of iterations is shown in Figure 6(a), and the solution is compared with that calculated by the fourth-order Runge-Kutta method (RK4) in Figure 6(b), and the difference is shown in Figure 6(c). Up to $t=1.92$ the solution fast tends to large value 21.22595, which causes the numerical solution obtained by the RK4 blowing up as shown in Figure 6(b) by dashed line. However, the GPS is also available up to $t=1.92$ as shown in Figure 6(b) by solid line.

We must emphasize that the results shown in (Adeyeye et al., 2015; Jaradat, 2008) are incorrect after $t=1.2$, whose errors are over 0.1 and more large up to 17.5 when time tends to $t=1.92$, where the GPS leads to the value 21.22595 .

\subsection{The Extension to Highly Nonlinear ODEs}

Through the above studies we can extend the Lie-group approach to highly nonlinear ODE:

$$
\frac{d y}{d t}=r(t)-2 q(t) y-F(y, t) y^{2}, y(0)=y_{0},
$$


where $F(y, t)$ may be a time-varying function of $t$ and nonlinear function of $y$.

Upon defining the integrating factor:

$$
u(t):=u(0) \exp \left[\int_{0}^{t}[q(\xi)+y(\xi) F(y(\xi), \xi)] d \xi\right]
$$

we can derive

$$
\frac{d}{d t}\left[\begin{array}{c}
u y \\
u
\end{array}\right]=\mathbf{A}\left[\begin{array}{c}
u y \\
u
\end{array}\right]
$$

where

$$
\mathbf{A}:=\left[\begin{array}{cc}
-q(t) & r(t) \\
F(y(t), t) & q(t)
\end{array}\right]
$$

Because the coefficient matrix A satisfies $\operatorname{tr} \mathbf{A}=0$, the resulting Lie-group is $S L(2, \mathbb{R})$, and thus the scheme in Section 8.2 can be extended to solve Eq. (80).

For example, let us consider (Murphy, 1960)

$$
\frac{d y}{d t}=\frac{2}{3 t} y+\frac{1}{3} y^{4}, y(1)=y_{0}
$$

which has an implicit analytical solution:

$$
y^{3}(t)\left(C+t^{3}\right)+3 t^{2}=0
$$

where $C=-1-3 / y_{0}^{3}$.

We fix $y_{0}=0.1$, and compute the solution of $y$ in a time interval of $t \in[1,10]$ by the GPS, where $h=0.001$ and $\varepsilon=10^{-10}$ were used. The number of iterations is shown in Figure 7(a), the solution is shown in Figure 7(b), and the error of $y^{3}$ is shown in Figure $7(\mathrm{c})$. It can be seen that the GPS provides very accurate solution with error smaller than $10^{-7}$.

\section{Conclusions}

The Riccati differential equation is known closely related to the second-order linear homogeneous differential equation through the variable transformation $y:=\dot{u} /(p u)$. We further explored that $u$ of the second-order linear homogeneous differential equation is really an integrating factor for the Riccati differential equation. Therefore, we proved that the above two equations share the same transformation group of $S L(2, \mathbb{R})$ as shown in Equations (16) and (23). Accordingly, we have developed a linear fraction scheme for the Riccati differential equation, whose coefficients matrix is an element of $S L(2, \mathbb{R})$. Applied this scheme to the logistic differential equation, we found that the group-preserving scheme has better accuracy and stability than other schemes compared in this paper. Several numerical examples were worked out. Upon comparing the schemes devised for the Riccati differential equation and second-order linear homogeneous and non-homogeneous differential equations to the corresponding closedform solutions, it was found that the group-preserving schemes all have accuracy better than $O\left(h^{2}\right)$. The extension of the $S L(2, \mathbb{R})$ Lie-group schemes to the Abel differential equation was made, of which the high performance was observed through three numerical tests. We also mentioned the extension to the higher-order nonlinear ODE by using the $S L(2, \mathbb{R})$ group-preserving scheme.

\section{Acknowledgements}

Highly appreciated are the project NSC-102-2221-E-002-125-MY3 and the 2011 Outstanding Research Award from the Ministry of Science and Technology of Taiwan, and the 2011 Taiwan Research Front Award and the 2014 ISI Highly Cited Researcher Award from Thomson Reuters. 


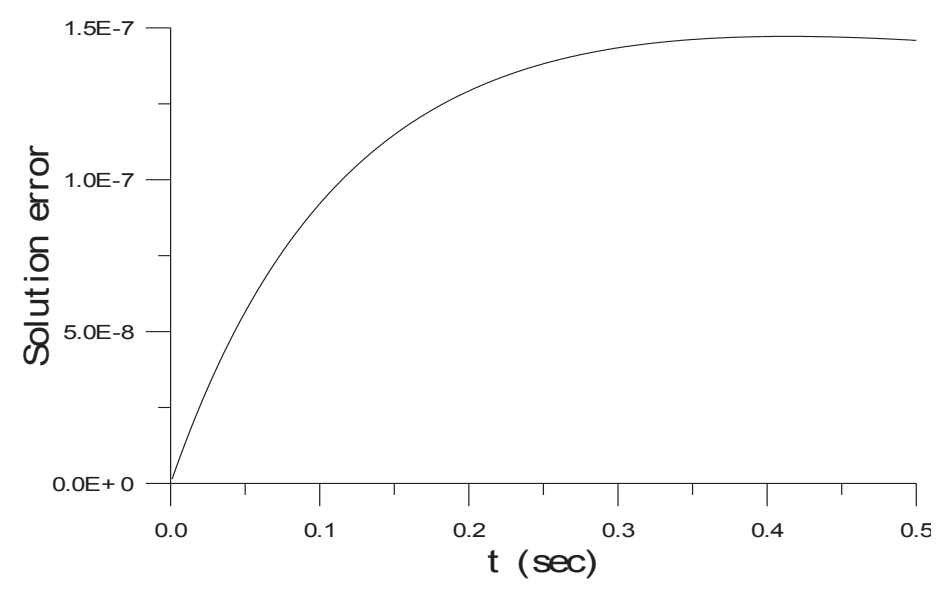

Figure 1. The solution error of the group-preserving scheme for a Riccati differential equation.
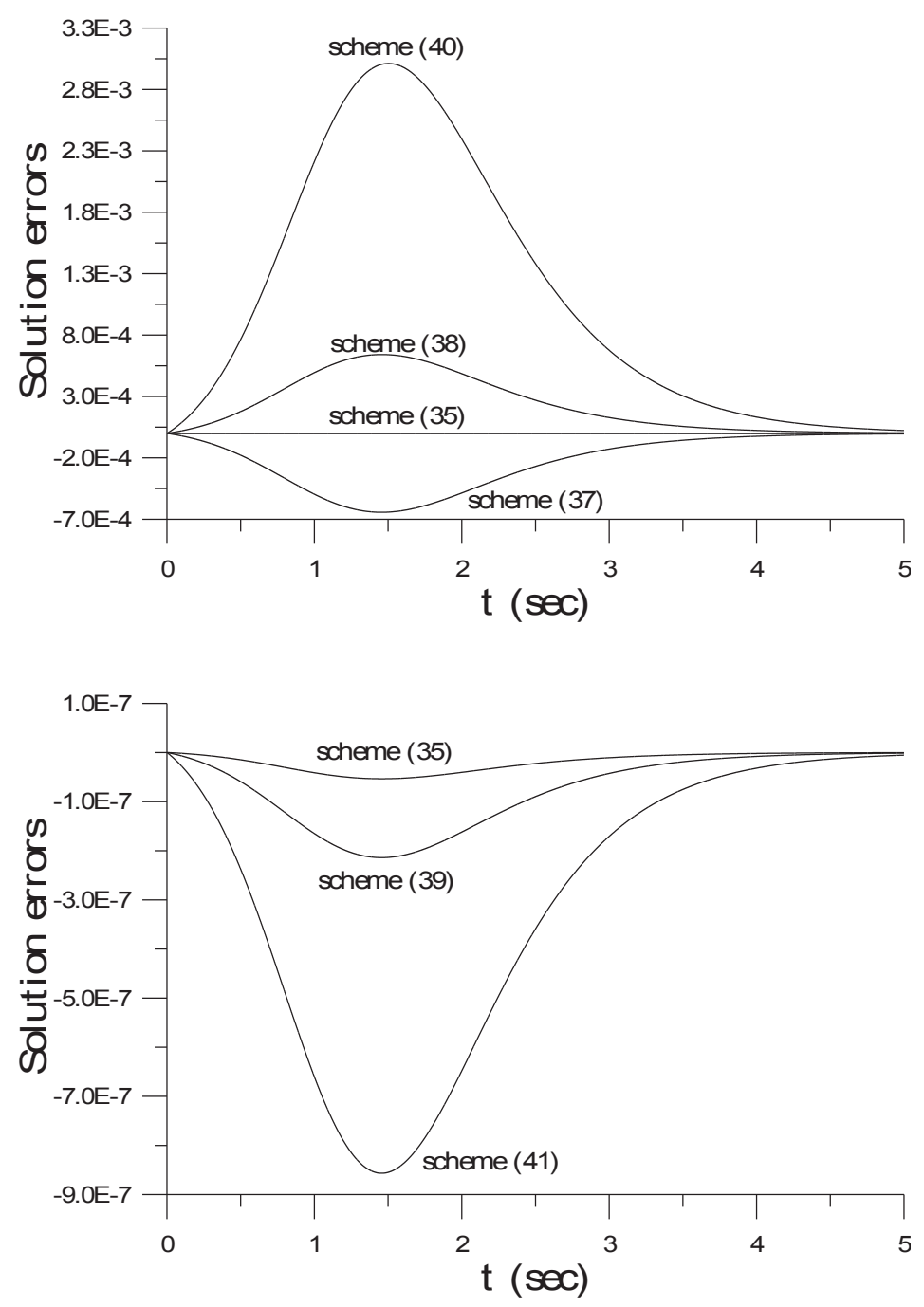

Figure 2. Comparing the solution errors of different numerical schemes used in the calculations for the logistic differential equation. 


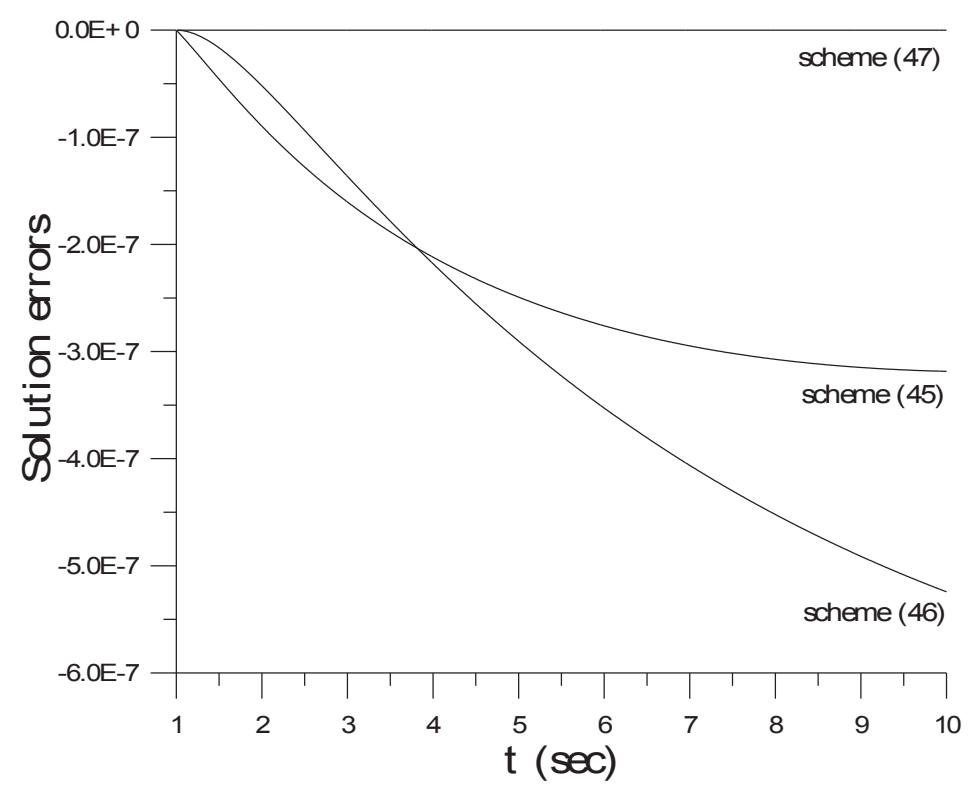

Figure 3. Comparing the solution errors of different numerical schemes used in the calculations for a second order linear homogeneous differential equation.

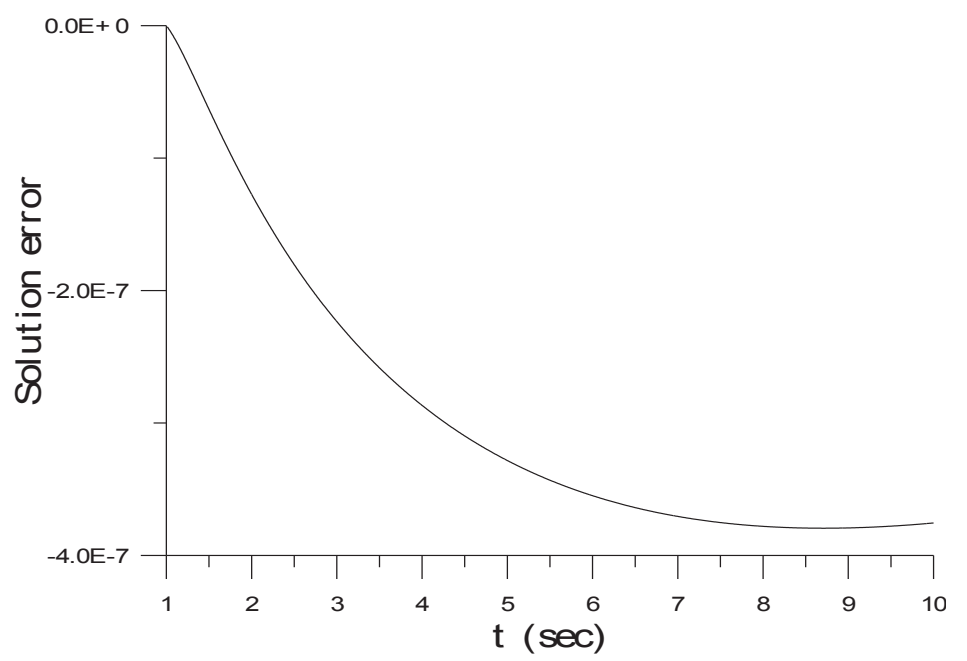

Figure 4. The solution error of the group-preserving scheme for a second order linear non-homogeneous differential equation. 

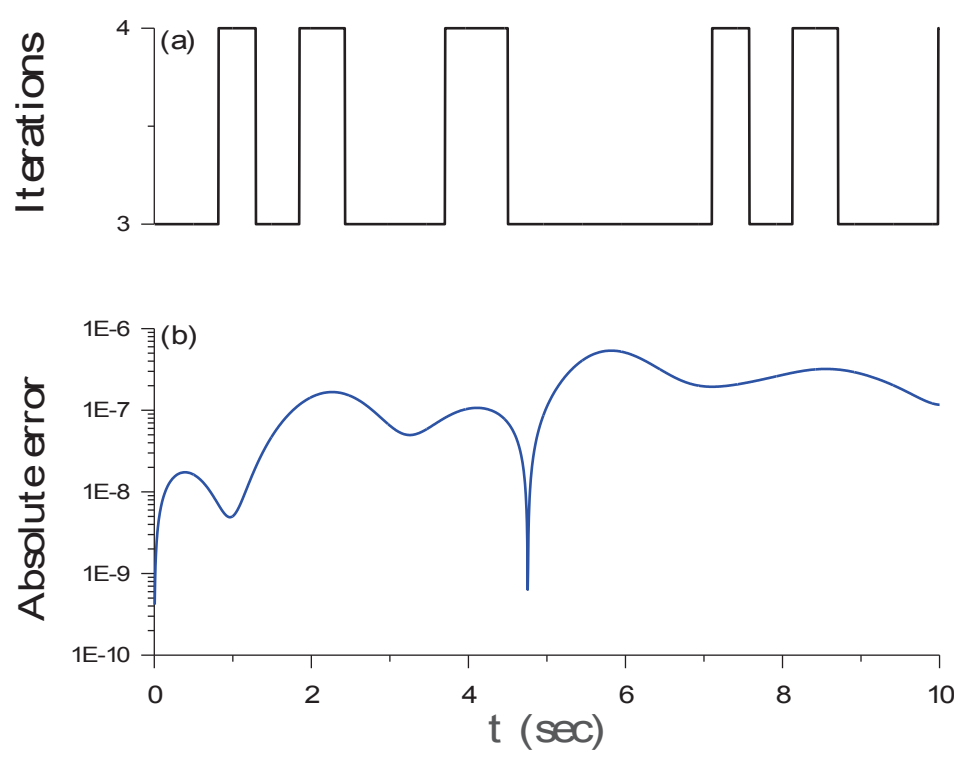

Figure 5. The number of iterations and solution error of the group-preserving scheme for Abel differential equation.
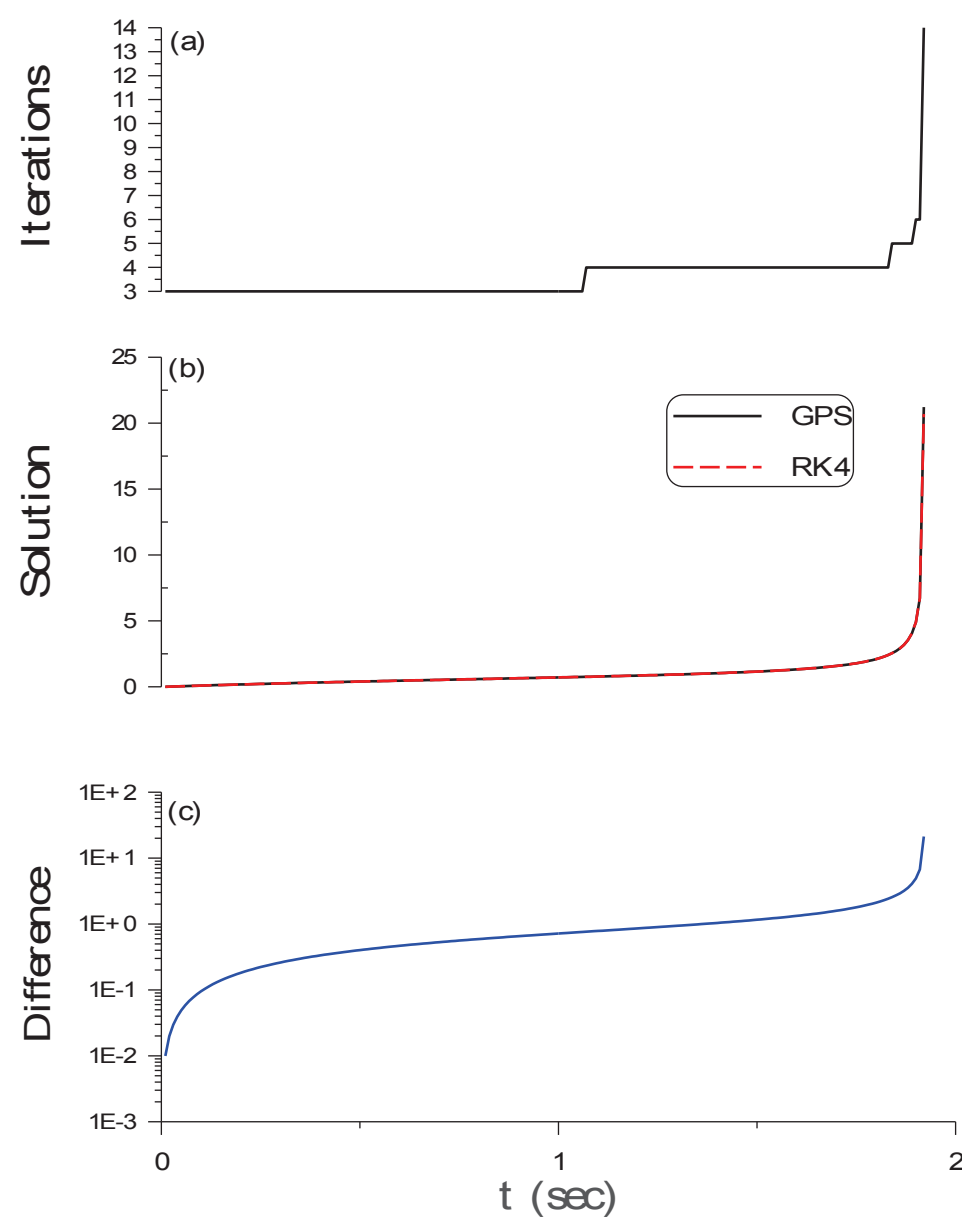

Figure 6. The number of iterations, solutions and solution error of the group-preserving scheme for Abel differential equation, upon compared with the RK4. 

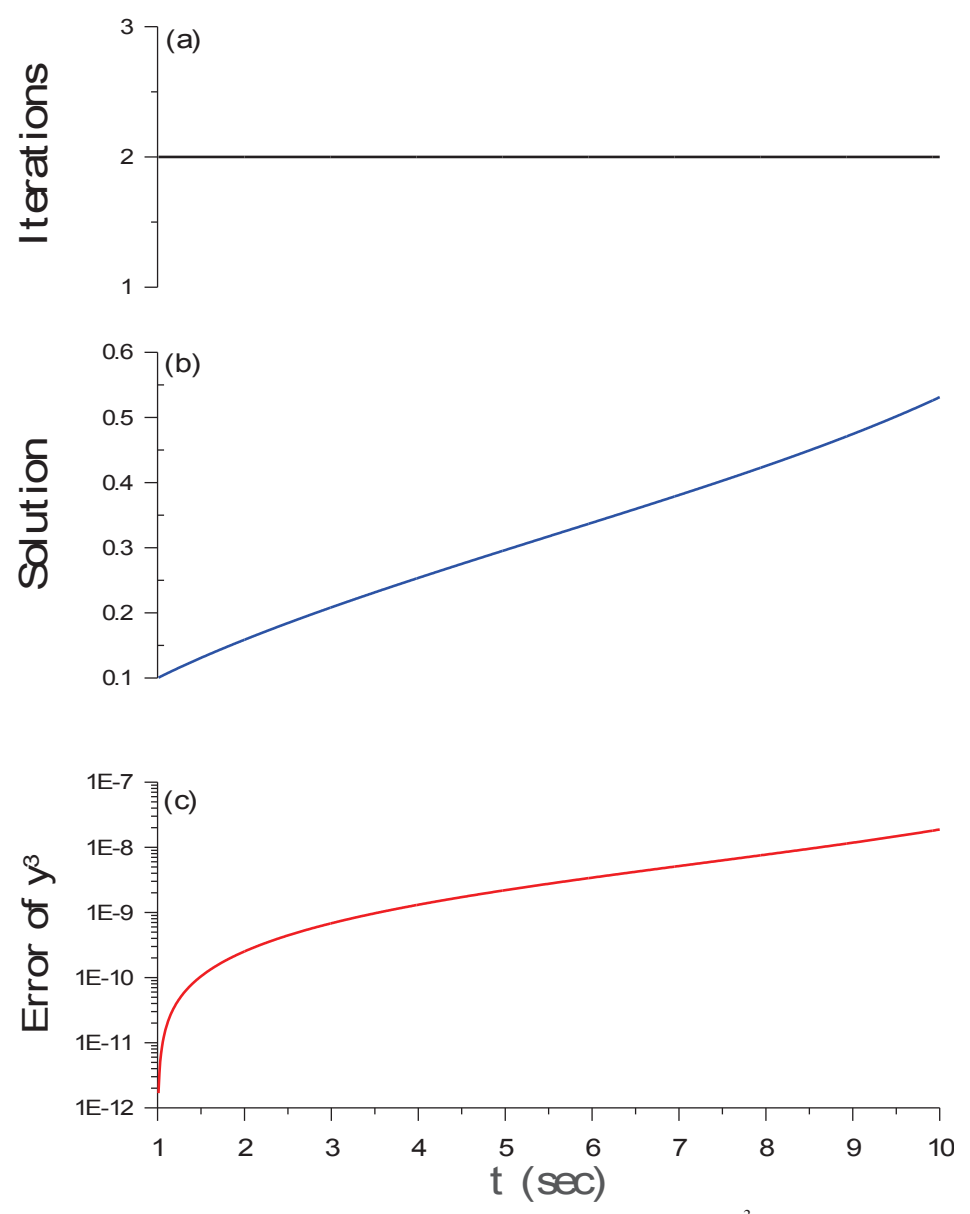

Figure 7. The number of iterations, solution and numerical error of $y^{3}$ of the group-preserving scheme for an quadruple nonlinear differential equation.

\section{References}

Adeyeye, F. J., Igobi, D. K., \& Ibijola, E. A. (2015). A new hybrid in the nonlinear part of Adomian decomposition method for initial value problem of ordinary differential equation. J. Math. Resea., 7, 102-109.

Chen, B., \& Solis, F. (1998). Discretizations of nonlinear differential equations using explicit finite order methods. J. Comp. Appl. Math., 90, 171-183. http://dx.doi.org/10.1016/S0377-0427(98)00017-X

Chen, R., Xu, Z., \& Sun, L. (1993). Finite-difference scheme to solve Schrödinger equations. Physical Review E, 47, 3799-3802. http://dx.doi.org/10.1103/PhysRevE.47.3799

Gander, M. J., \& Meyer-Spasche, R. (2000). An introduction to numerical integrators preserving physical properties. In Applications of Nonstandard Finite Difference Schemes, R. E. Mickens ed., World Scientific, Singapore. http://dx.doi.org/10.1142/9789812813251_0005

Hashemi, M. S. (2015). Constructing a new geometric numerical integration method to the nonlinear heat transfer equations. Commun. Nonl. Sci. Numer. Simul., 22, 990-1001. http://dx.doi.org/10.1016/j.cnsns.2014.09.026

Hochbruck, M., \& Ostermann, A. (2010). Exponential integrators. Acta Numerica, 19, 209-286. http://dx.doi.org/10.1017/S0962492910000048

Iserles, A., Munthe-Kaas, H. Z., Nørsett, S. P., \& Zanna, A. (2000). Lie-group methods. Acta Numerica, 9 , 215-365. http://dx.doi.org/10.1017/S0962492900002154

Jaradat, O. K. (2008). Adomian decomposition method for solving Abelian differential equations. J. Appl. Sci., 8 , 1962-1966.

Liu, C.-S. (2001). Cone of non-linear dynamical system and group preserving schemes. Int. J. Non-Linear Mech., 
36, 1047-1068. http://dx.doi.org/10.1016/S0020-7462(00)00069-X

Liu, C.-S. (2006). Preserving constraints of differential equations by numerical methods based on integrating factors. CMES: Comput. Model. Eng. Sci., 12, 83-107.

Liu, C.-S. (2007). New integrating methods for time-varying linear systems and Lie-group computations. CMES: Comput. Model. Eng. Sci., 20, 157-175.

Liu, C.-S. (2012). Computing the eigenvalues of the generalized Sturm-Liouville problems based on the Lie-group S L(2, $\mathbb{R})$. J. Comput. Appl. Math., 236, 4547-4560. http://dx.doi.org/10.1016/j.cam.2012.05.006

Liu, C.-S. (2013a). Developing an $S L(2, \mathbb{R})$ Lie-group shooting method for a singular $\phi$-Laplacian in a nonlinear ODE. Commun. Nonl. Sci. Numer. Simul., 18, 2327-2339. http://dx.doi.org/10.1016/j.cnsns.2012.12.021

Liu, C.-S. (2013b). A method of Lie-symmetry $G L(n, \mathbb{R})$ for solving non-linear dynamical systems. Int. J. NonLinear Mech., 52, 85-95. http://dx.doi.org/10.1016/j.ijnonlinmec.2013.01.015

Liu, C.-S. (2015). A novel Lie-group theory and complexity of nonlinear dynamical systems. Commun. Nonl. Sci. Numer. Simul., 20, 39-58. http://dx.doi.org/10.1016/j.cnsns.2014.05.004

Mickens, R. E. (1999). Discretizations of nonlinear differential equations using explicit nonstandard methods. $J$. Comp. Appl. Math., 110, 181-185. http://dx.doi.org/10.1016/S0377-0427(99)00233-2

Mickens, R. E. (2000). Nonstandard Finite Difference Schemes. In Applications of Nonstandard Finite Difference Schemes, R. E. Mickens ed., World Scientific, Singapore.

Mickens, R. E., \& Ramadhani, I. (1992). Finite-difference scheme for the numerical solution of the Schrödinger equation. Physical Review A, 45, 2074-2075.

Munthe-Kaas, H. (1999). High order Runge-Kutta methods on manifolds. Appl. Numer. Math., 29, 115-127. http://dx.doi.org/10.1016/S0168-9274(98)00030-0

Murphy, G. M. (1960). Ordinary Differential Equations and Their Solutions. Litton Educational Publishing Inc., New York.

Sachdev, P. L. (1991). Nonlinear Ordinary Differential Equations and Their Applications. Marcel Dekker Inc., New York.

Wang, Y., Twizell, E. H., \& Price, W. G. (1992). Second order numerical methods for the solution of equations in population modelling. Comm. Appl. Numer. Meth., 8, 511-518.

\section{Copyrights}

Copyright for this article is retained by the author(s), with first publication rights granted to the journal.

This is an open-access article distributed under the terms and conditions of the Creative Commons Attribution license (http://creativecommons.org/licenses/by/3.0/). 